\title{
Research on modelling and scheduling strategy for mine transportation control system based on CPS
}

\section{Jingzhao Li, Dayu Yang* and Xiaoming Zhang}

College of Electrical and Information Engineering,

Anhui University of Science and Technology,

Huainan, 232001, China

Email: jzhli@aust.edu.cn

Email: zyzprime@gmail.com

Email: zxm_aust@126.com

*Corresponding author

\begin{abstract}
Cyber-physical systems (CPS) have made great strides in industrial control, intelligent transportation, remote medical and other fields. Coal mine transportation control system is a representative multi-subsystem of CPS. In this study, for the sake of optimising the scheduling mechanism for event response and precise control of tramcar behaviour in the system, we propose an event-orient scheduling algorithm (EOSA) to achieve a rapid response based on building a no-memory continuous time model. Therefore, the control system can match tasks in the queue according to the real-time load situation, and each physical entity can accurately execute the instruction under discrete event environment. Simulation results have shown that the proposed algorithm has a higher execution speed compared with the hybrid genetic algorithm and fuzzy clustering scheduling algorithm. Our approach realises the load balancing of global task scheduling and is more suitable for mine transportation scenario.
\end{abstract}

Keywords: cyber-physical systems; CPS; mathematical model construction; scheduling strategy; priority queuing; mine transportation.

Reference to this paper should be made as follows: Li, J., Yang, D. and Zhang, X. (2019) 'Research on modelling and scheduling strategy for mine transportation control system based on CPS', Int. J. Embedded Systems, Vol. 11, No. 5, pp.678-686.

Biographical notes: Jingzhao Li received his MA degree from China University of Mine and Technology in 1992, and PhD degree from the Key Lab of Power Electronics and Power Drives at Hefei University of Science and Technology, in 2003. He is currently a Professor at College of Electrical and Information Engineering, Anhui University of Science and Technology, China. His research interests include computer control, internet of things technology and embedded systems.

Dayu Yang is a PhD candidate at the School of Electrical and Information Engineering, Anhui University of Science and Technology, China. He received his Bachelor degree in Communication Engineering from Huaibei Normal University in 2013. His current research interests include cyber physical systems and internet of things.

Xiaoming Zhang received his Bachelor degree in Electrical Engineering from Anhui University of Science and Technology (AUST), Anhui, Huainan in 2015. He is currently a PhD candidate at AUST. His current research interests are mobile communication and wireless sensor networks.

\section{Introduction}

Cyber-physical systems (CPS) are an intelligent system that integrates information processing technology and the physical environment, which achieved embedded computer and network deep integration. It has various characteristics such as deep embedding, ubiquitous interconnection, intelligent perception and collaboration (Muller, 2017; Akkaya et al., 2016; Isasa et al., 2017). As a pillar economy in China, coal mine enterprises need to keep up with the pace of industrialisation and informatisation. Due to using its own dedicated communication protocol for each substation, the traditional coal mine safety monitoring system has poor compatibility and priority support (Akkaş,
2016), which cannot meet a rapid response to emergencies and processing timely. To accomplish complicated industrial control tasks, it is necessary to construct a distributed hierarchical model that can be suitable with industrial Ethernet, various fieldbus substations and equipments. Inclined roadway transportation control system is an important part of the coal mine daily production, which is the main route for transport coal, equipment, and stuff in the underground mining area. During the operation of the tramcar, the staff can only drive by voice signal so they could not know the exact situation in the tunnel, which leads to safety accidents such as tramcar falloff rails, roll-over or people wounding. 
In the current distributed computing environment, the concept of CPS provides an effective solution to solve the parallel job scheduling and improve the efficiency of operation. However, in order to enable the control system as much as possible to complete the task within the specified period, an excellent and reliable scheduling strategy with appropriate resource management must be established. Inclined roadway transportation control system is a vital part of coal mining daily production, so it is necessary to put the job queue on the resource node that can meet the task computing capability requirements and the resources are fully utilised.

The rest of this paper is organised as follows. In Section 2, we first introduce recent related works of CPS task scheduling and theoretical model construction. Next, in Section 3, based on the analysis of the structure of CPS, we build a non-memory continuous time model of inclined lane transportation control system. In Section 4, by analysing the status parameters of the transportation control system, the fixpoint semantic model and an event-oriented scheduling algorithm is proposed. In order to verify the performance of the proposed algorithm, we describe the implementation method and a comparative analysis in Section 5. Finally, we conclude the contribution of this paper in Section 6.

\section{Related works}

In the aspect of pragmatic modelling of CPS, previous studies have primarily concentrated on modelling under different application scenarios, by establishing the relationship between physical entities and information space to achieve the deep interaction between physical and calculation process.

\subsection{CPS in transportation}

Since the traditional transportation systems cannot achieve full coordination and optimisation due to the lack of widespread interconnection, intercommunication, and interoperability. Lin et al. (2016) discussed the concept of CPS based on event semantics and described the determination states of traffic, the proposed dynamic Enroute real-time route guidance (DEDR) scheme can effectively mitigate road congestion and increase traffic efficiency. In terms of CPS structure, Liu et al. (2016) believed that T-CPS is mainly composed of corresponding control software, communication network, and physical devices, and proposed a control strategy based on lattice hydrodynamic model to achieve system reliability, accuracy and effectiveness automatic control. In unmanned ground vehicle scenario, Lee and Kang (2017) proposed a periodic, fault-tolerant CPS task assignment model, which improved the effectiveness and stability of the underlying physical subsystem and established a priority assignment mechanism. The allocation strategy significantly reduces the operating costs of the system. How to predict the traffic flow of the vehicle CPS correctly, Chen et al. (2018) proposed a method based on fuzzy deep-learning methods (FDCN), by establishing a fuzzy deep convolutional neural network and analysing the temporal and spatial correlation of traffic flow, the impact of uncertain data ultimately reduced.

\subsection{CPS in healthcare}

Medical CPS is one of the important applications of healthcare service field, which provides an effective solution for improving medical services. Plaza et al. (2018) made a review of recent medical CPS applications, pointing out that medical CPS is an organic integration and deep collaboration of $3 \mathrm{C}$ (computation, communication, control) technology, the functional and non-functional characteristics, quality attribute architecture views were both discussed at the same time. Furthermore, Rao et al. (2018) studied medical CPS framework and proposed a safety verification method based on differential-algebraic dynamic logic, which implements hierarchical verification of attributes in the system. A patient-centred health-CPS for telemedicine services was designed by Zhang et al. (2017), the system realises distributed storage and parallel processing of data, the treatment plan can dispatch relevant units to provide efficient medical services in the fastest time.

\subsection{CPS in military services}

In the military services, information resources are very important, military cyber-physical systems (MCPS) as a dedicated application system emphasise the real-time and dynamic characteristics of information space. Unmanned aerial vehicles (UAV) are a typical example of embedded CPS, Gonçalves et al. (2016) proposed two behavioural models with event-oriented state refinement and made a collaborative simulation, which provided a new method for the system-integrated UAV design process. In response to security and privacy issues in control system, Sadreazami et al. (2018) applied the CPS methods to construct security framework for network control system (NCS) and proposed a solution for adaptive structure-aware monitory, the Bayesian conditional probability theory is used to correlate the continuous detection at the same time, which effectively reduces the false alarm rate of intrusion detection.

\subsection{CPS in other fields}

According to the characteristics of distributed resource and dynamic changes of the next generation smart grid, You et al. (2017) applied the concept of CPS into complex power 
systems, a dynamic mathematical model of distributed task decisions based on the network is proposed. By analysing the resource characteristics of CPS, Bessani et al. (2016) analyse the reliability problems in the cyber-physical power distribution system (CPPDS). Based on the analysis of system response time and decision quality, a sequential Monte Carlo simulation method is proposed, which corrects the response time and improves the reliability of CPPDS. In order to improve the efficiency of the CPU power of the cloud data centre and maintain the activity of the entire network system, Rossi et al. (2017) abstract the cloud centre device as a physical node, and determine the energy acquired by the CPU while performing different tasks, and make a qualitative description of system reliability, then proposed a corresponding management algorithms to maximise the efficiency of device execution. A novel obstacle avoidance scheme based on CPS for networked surveillance robots is presented in Khan et al. (2016), the robots in the system are no longer single embedded devices, they not only have the ability to sense and transmit information, but also have intelligent judgements on information world and feedback control to the physical world. Aiming at the problem of integrating a large number of heterogeneous sensors into the information system and operating each other seamlessly in the precision agriculture monitoring, Chen et al. (2015) proposed the CPS architecture of service-based integrated open geospatial and realised the design of service-oriented information exchange between heterogeneous physical sensors and different clients.

These articles mainly focus on the semantic modelling of CPS in specific application scenarios, involving intelligent transportation, healthcare, military services, and other fields. Significant progress has been made in system resource optimisation, which has very important theoretical significance and engineering application value.

\section{The architecture of cyber-physical systems}

CPS mainly consists of three parts: a physical entity, computing platform, and network communication module. Physical entity is the physical part of CPS, which refers to the real and visible entity. Computing platform is the way to achieve the calculation process in CPS that is composed of sensors, drivers and related embedded devices. Network communication module provides a communication mechanism of computer and is the interface between physical and information world. As is shown in Figure 1, two computing platforms $\mathrm{A}$ and $\mathrm{B}$ are connected to each other, and each platform has its own sensor and corresponding embedded devices. Information of the surrounding environment is perceived and collected by the relevant sensor, and then through the embedded device-related control rules after the code, and ultimately achieves precise control of physical entities.
Figure 1 Fundamental architecture for CPS (see online version for colours)

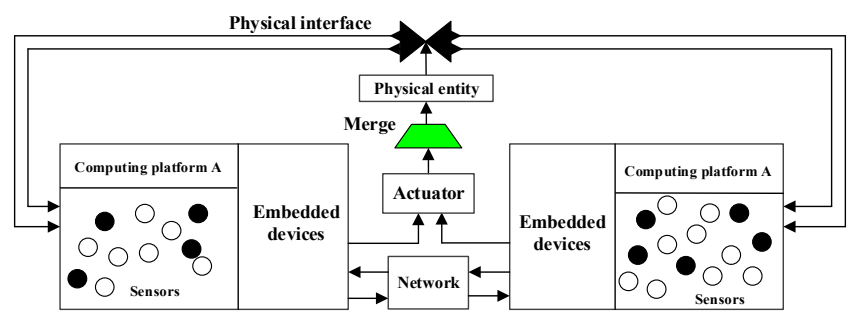

\subsection{Main characteristics of CPS}

While emphasising the perception, communication, and calculation of physical world, CPS has increased the ability to interact with the physical entities and its surrounding environment, which is a combination of the Internet of Things (IOT) and system control technology. After making a rigorous system-level scientific division based on multisource data modelling, the relationship between virtual and physical systems is established, and the decision layer is continuously optimised to achieve global collaborative optimisation at the same time. Therefore, the main characteristics of CPS are summarised as follows:

1 Intelligent perception: physical entities need to sense the external environment and collect the necessary information continuously during the production. Considering the basic requirement of safe production, intelligent perception tends to emphasise the choice of physical entities with a wide range of spatial and sustained time requirements. For example, the monitoring and control of tramcar running status have certain continuity in space and time distribution.

2 Real-time feedback: when some relative information is sensed and collected by physical entities, the current target needs to make a feedback in real time. As a result, the system instructions will be corrected according to the difference between the expected behaviour of the physical object, then the actual behaviour of the system can be corrected to ensure the real-time, continuity and reliability of information transmission.

3 Ubiquitous connection: network communication is the basic guarantee of CPS, which can achieve interoperability between CPS internal and other external units. In coal mine CPS, there is more emphasis to build low-delay, low-power, highreliability network mode in a heterogeneous environment.

4 Mapping for virtual and reality: by constructing a closed-loop data channel between information and the physical world, CPS implements the interaction between virtual and reality. Besides, by dynamically tracking the working status of entities (i.e., the result of measurement, retroactive information, etc.), the static model with perception, analysis, and decision-making capabilities can be reconstructed in the information 
space. Effective decisions can be formed in response to the complex environment, and ultimately applied to entities. Finally, the limited resources are optimally allocated.

5 Scientific decision making: with the help of relevant scientific methods, these accumulated data can be used to analyse, assess reality and forecast the future. Furthermore, the optimal decision will be made in accordance with certain constraints of time, and all of the acquired information from different systems are weighted to form the optimal decision to control entities in physical space, which is the core section of CPS.

6 Precise execution: the final decisions that formed in the information space will be applied to entities in the physical space in various ways. Besides, the related entities can only make decisions through the form of control information. The essence of execution is to transform the decisions into executable commands and implement them at the physical level to obtain more optimised data. Therefore, the operation of equipment will become more reliable, the scheduling of system resource will be more reasonable and enterprise operating more efficiently, the intelligent devices of physical space will work collaboratively to achieve global optimisation.

\subsection{Architecture control unit of cyber-physical systems}

A typical structure of the CPS control system is shown in Figure 2. The controller acts on the object through the instruction of actuator. However, some uncontrollable factors from the environment may also affect the controlled object ( $\mathrm{Gu}$ et al., 2016). The input command from the controlled object is used as a reference for the output response, and the corresponding decision is made according to the data obtained from the sensors.

Figure 2 A typical architecture of CPS control system

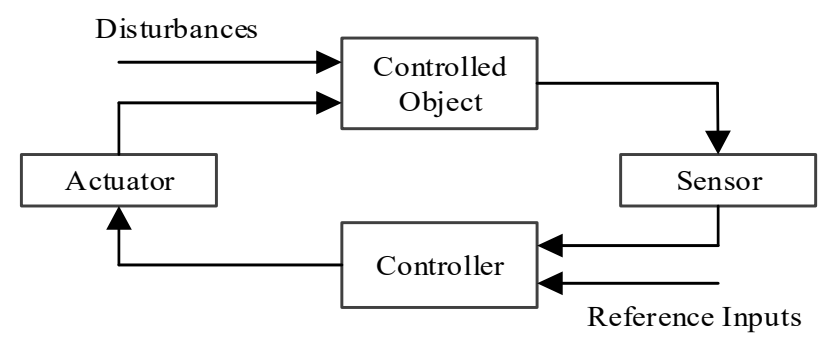

\subsection{Construction of non-memory continuous time model for inclined lane transportation control system}

During the actual production and transportation process of mine, inclined lane transportation system mainly including signal system, anti-slip tramcar system, and frequency control system, all of them are continuous-time systems.
Therefore, the continuous dynamic behaviour of inclined lane transportation process can be modelled by ordinary differential equations (ODE). As shown in Figure 3, the inclined lane transportation control system is selected as the research object, the upper working face is defined as level $n$, the lower is level $n-1$, and the middle part is inclined lane, which can perform various automatic operation modes, such as tramcar blocked (stop), run and fault under the different external conditions.

Figure 3 Model switching for transportation control system

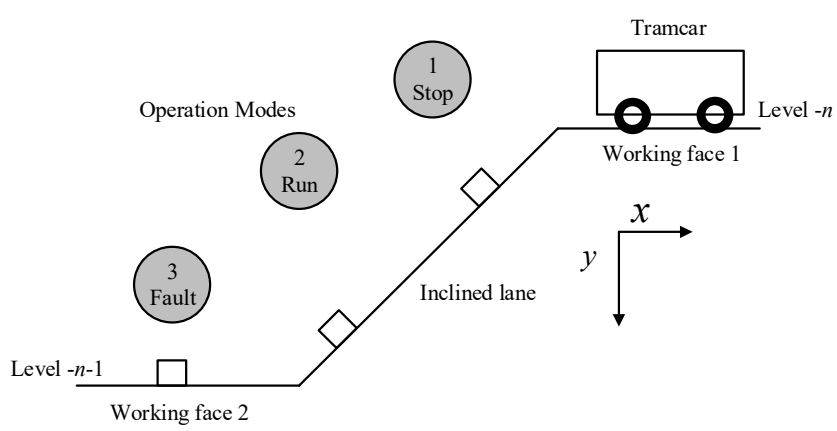

If the output of a system only depends on current input parameters and has no connection with the past input, then the system is called a non-memory system (Li et al., 2017). As shown in Figure 4, the parametric model can be described as follows.

Figure 4 A continuous time system model with $n$ inputs, $m$ outputs (see online version for colours)

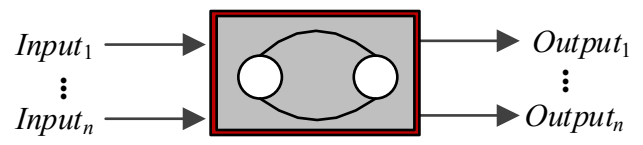

For a continuous no-memory time system $S: X \rightarrow Y$, and $X=A^{\mathbb{R}}, \quad Y=B^{\mathbb{R}}, A$ they $B$ were both the state set of system, existing a function: $F: A \rightarrow B$, and for $\left(x_{1}, x_{2}, \ldots\right.$, $\left.x_{n}\right) \in X,\left.x\right|_{t \leq \tau}$ represents a time constraint function, when $t \leq \tau$, exist $\left.x\right|_{t \leq r}(t)=x(t)$, we can get:

$$
Y(t)=(S M \quad p, q(X(t)) \quad(t))=F(X(t))
$$

As shown in Figure 5, the system status signal character synchronous-reactive (SR) model with four state control signals is established, among them, $f_{0}(n), f_{1}(n), f_{2}(n), f_{3}(n)$ respectively represents: start, normal operation, emergency block and stop. $n$ represents the time interval at a certain moment. For example, the first time interval can be defined as: $n=0$, the second time interval is $n=1$, and so on. Each character performs from input to output function. Such as the start status executive function $f_{1}(0)$, when $n=0$, that is the input value $S_{0}(0)$ of $P_{3}$, it will produce an output value $S_{1}(0)=\left(f_{1}(0) S_{0}(0)\right)$.

Because any synchronous reactive model can be rearranged into a single role model of the function $f(n)$ at $n$ a time. As shown in Figure 6, the domain of the signal function can be regarded as one tuple

$$
S(n)=\left(S_{0}(n), S_{1}(n), \ldots, S_{5}(n)\right)
$$


Figure 5 Synchronous reactive model of mine transportation system

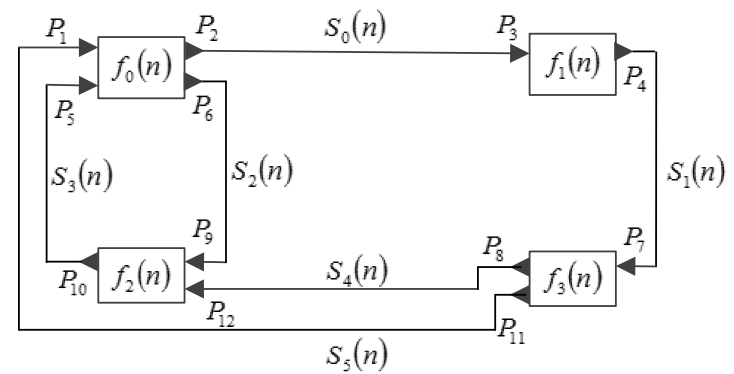

Figure 6 Simplified synchronous reactive model

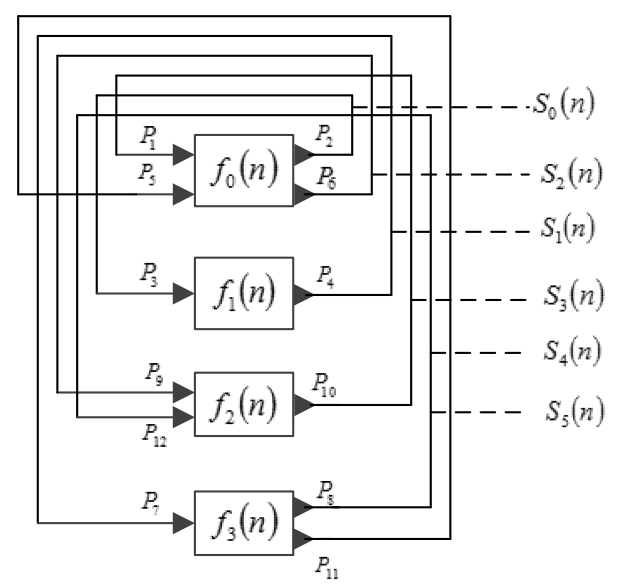

\section{Multi-task scheduling algorithm for mine transportation control system}

\subsection{Establishment of related parameters for system status}

Control system is an important part of inclined lane transportation system, as shown in Table 1, the system has six operating states during actual operation, that is idle state $S_{0}$ represents control system is power off, and there is no task to be carried out. $S_{1}$ represents the system is in the blocked status, tramcar stop running. $S_{2}$ represents the device of blocker closure, the system is in operating status. $S_{3}$ represents alert status, and execute the alarm task. When the control system did not receive the feedback signal from tramcar blocker, the system will be in fault status $S_{4}$, and $S_{5}$ represents record status of the system.

Table 1a Related parameters of system status signal

\begin{tabular}{lccc}
\hline Status label & $S_{0}$ & $S_{1}$ & $S_{2}$ \\
\hline System status & Idle & Block & Run \\
Executive task & Wait & Lift & Closure \\
\hline
\end{tabular}

Table 1b Related parameters of system status signal

\begin{tabular}{lccc}
\hline Status label & $S_{3}$ & $S_{4}$ & $S_{5}$ \\
\hline System status & Alert & Fault & Run \\
Executive task & Alarm & Process & Record \\
\hline
\end{tabular}

\subsection{Computing model for a control system based on directed acyclic graph}

The direct cause of control system operation is the generation of the external trigger (Fang et al., 2018). As shown in Table 2, the external trigger events can be summarised as follows: closure, run and fault. These events all have non-memory characteristics, that is, the execution unit of the system will only make corresponding operations in terms of the current events.

Table 2 Trigger event of a transportation control system

\begin{tabular}{lc}
\hline Label & Trigger event \\
\hline$e_{1}$ & Block \\
$e_{2}$ & Run \\
$e_{3}$ & Fault \\
\hline
\end{tabular}

According to the relevant definition of directed acyclic graph (DAG) theory (Liu et al., 2017), combined with trigger event parameters of system status in Section 4.1, we can establish a mathematical model for inclined lane transportation system $M=M\left(A, S, Z, S_{0}, f, g\right)$, therefore, the input set $A$, status set $S$, and output set $Z$ are expressed as follows:

$$
\begin{aligned}
A & =\left\{e_{1}, e_{2}, e_{3}\right\}, \\
S & =\left\{S_{0}, S_{1}, S_{2}, S_{3}, S_{4}, S_{5}\right\}, \\
Z & =\{x, y, z\}
\end{aligned}
$$

Among them, $S_{0}$ is the initial status of the system, $x, y, z$ represents output set respectively: stop, run and record. The status diagram of the control system $D=D(M)$ can be illustrated as Figure 7.

Figure 7 Status transition diagram for control system

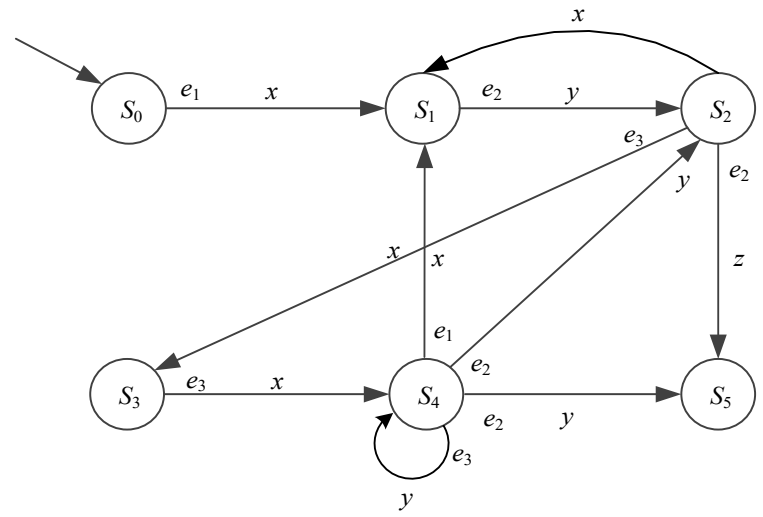


Combined with the next state function $f$ and output function $g$ into the same table, we can get the function $F: S \times A \rightarrow S$ $\times Z$ and defined as follows:

$$
F\left(S_{i}, e_{j}\right)=\left[f\left(S_{i}, e_{j}\right), g\left(S_{i}, e_{j}\right)\right]
$$

Referring to Figure 7, the state table of $M$ can be obtained and the combination of the table is expressed as: $\left(S_{k}, t_{r}\right)$. $S_{k}=f\left(S_{i}, e_{j}\right)$ represent the next status of trigger event that is all vertices in Figure 7. $t_{r}=g\left(S_{i}, e_{j}\right)$ is the output symbol. For instance, when $w=e_{1} e_{2} e_{3} e_{3} e_{2} e_{2}$ is the input word of the system at a certain moment, in the initial status $S_{0}$, the status sequence and the output word can be obtained respectively: $u=S_{0} S_{1} S_{2} S_{3} S_{4} S_{2} S_{5}, r=x y x x y x y z$.

Table 3 Status table for mine transportation control system

\begin{tabular}{lccc}
\hline$F$ & $e_{1}$ & $e_{2}$ & $e_{3}$ \\
\hline$S_{0}$ & $S_{1}, x$ & - & - \\
$S_{1}$ & - & $S_{2}, y$ & - \\
$S_{2}$ & - & $S_{5}, z$ & $S_{3}, x$ \\
$S_{3}$ & - & - & $S_{4}, x$ \\
$S_{4}$ & $S_{1}, x$ & $S_{2}, y$ & $S_{4}, y$ \\
$S_{5}$ & - & - & - \\
\hline
\end{tabular}

\subsection{Performance evaluation index and designation for scheduling strategy}

The goal of scheduling strategy is to meet the needs of end physical entities as much as possible while maximising the utilisation of system computing resources (Nayak et al., 2016; Digalwar et al., 2017; Jindai and Bedi, 2018). In order to value the status transitions of embedded control system actuators, the finite state machine of coal mine inclined lane transportation control system can be established as follows:

$$
M=M\left(A, S, Z, S_{0}, f, g\right)
$$

Let the probability output of the event $e_{i}$ by the actuator is $p\left(e_{i}\right)$, then the free information quantity $I$ can be expressed:

$$
I\left(e_{i}\right)=-l b\left(p\left(e_{i}\right)\right)=l b\left(1 / p\left(e_{i}\right)\right)
$$

Because $I\left(e_{i}\right)$ is a strictly decreasing function in domain $[0,1]$, when $p\left(e_{1}\right)<p\left(e_{2}\right), I\left(e_{1}\right)>I\left(e_{2}\right)$. It represents the smaller occurrence probability of event $e_{1}$, the greater uncertainty than $e_{2}$. Specially, when $p\left(e_{i}\right)=0, I\left(e_{i}\right) \rightarrow \infty$, and $p\left(e_{i}\right)=1, I\left(e_{i}\right)=0$.

Let random variable $X$ and the corresponding probabilities are called probability space:

$$
\left[\begin{array}{c}
A \\
P(A)
\end{array}\right]=\left[\begin{array}{cccc}
A=e_{1} & A=e_{2} & \cdots & A=e_{i} \\
p\left(e_{1}\right) & p\left(e_{2}\right) & \cdots & p\left(e_{i}\right)
\end{array}\right]
$$

Among it, $P(A)=\left[0 \leq p\left(e_{i}\right) \leq 1 \cup \sum_{j=0}^{i} p\left(e_{i}\right)=1\right]$.

Assuming the system performs tasks without external disturbances, the actuator's uncertainty can be represented by the average free information quantity $H$.

$$
H[p(A)]=E\left\{p\left[I\left(e_{i}\right)\right]\right\}=-\sum_{j=1}^{i}\left[p\left(e_{j}\right) l b\left(p\left(e_{j}\right)\right)\right]
$$

According to information entropy theory, $H[(p(A))]$ has the following properties:

$1 \quad H\left[p\left(e_{1}\right), p\left(e_{2}\right), \ldots, p\left(e_{i}\right)\right]=H\left[p\left(e_{1}\right), p\left(e_{i}\right), \ldots, p\left(e_{2}\right)\right]$

The changed sequence of each event does not affect the value of the information entropy.

$2 H\left[p(A)=H\left[p\left(e_{1}\right), p\left(e_{2}\right), \ldots, p\left(e_{i}\right)\right] \geq 0\right.$

Only if the actuator is a define source of information, the equality holds.

$3 \lim _{b \rightarrow 0} H_{i+1}\left[p\left(e_{1}\right), \ldots, p\left(e_{i}\right), p(b)\right]=H_{i}\left[p\left(e_{1}\right), \ldots, p\left(e_{i}\right)\right]$

That is, when a small probability event occurred, it will not change the entropy of the information source.

$4 H\left[p\left(e_{1}\right), p\left(e_{2}\right), \ldots, p\left(e_{i}\right) \leq H(1 / i, 1 / i, \ldots, 1 / i)=l b(i)\right.$

When the probability of event equal occurred, $H[p(A)]$ get the local maximum. Besides, in the domain $[0,1]$, the value of $H[p(A)]$ is global maximum, that is, $H$ is a continuous function.

Let the evaluation strategy be $V$, data quality is $Q$, we can get:

$$
V_{A}(t)=\alpha(k-n) H(A)+\beta Q(t)
$$

Among it, $\alpha$ and $\beta$ are both evaluation parameters, $\alpha, \beta \geq 1$, $(k-n)$ is the time signal period of measured event information entropy.

$$
\begin{aligned}
& H(A)=l b(i) \times(1-\chi)=-\sum_{j=0}^{i}\left[p\left(a_{j}\right) l b\left(p\left(a_{j}\right)\right)\right] \\
& Q(t)=1-\left(t_{r}+T_{V}^{\max }-T_{v}^{\min }\right) /\left(T_{V}^{\max }-T_{V}^{\min }\right)
\end{aligned}
$$

Let $f_{1}=\alpha(k-n) H(A), f_{2} \beta Q(t)$, then we can get:

$$
V_{A}(t)=F(A)=\sum_{j=1}^{2}\left(\xi_{j} \times f_{f}\right)
$$

$\xi_{j}=[0,1]$ is a random weighting factor, and $\sum_{j=1}^{2} \xi_{j}=1$. Considering the dominant position of $H(A)$ and $Q(t)$, in function $F(A)$, if $f_{1}<f_{2}$, we observe get:

$$
\left[\forall t \in C, f_{1} \leq f_{2}\right] \wedge\left[\exists t \in C, f_{1}<f_{2}\right]
$$

According to the algorithm design principle of flat model semantics, combined with the defined parameters of a control system model in Section 4.2, the execution algorithm for scheduling control strategy is can be illustrated as follows: 
Algorithm 1 Scheduling control strategy execution algorithm

Input: Tramcar transportation sequence $Q$, initial event set $A$

Output: Output control strategy set $Z$

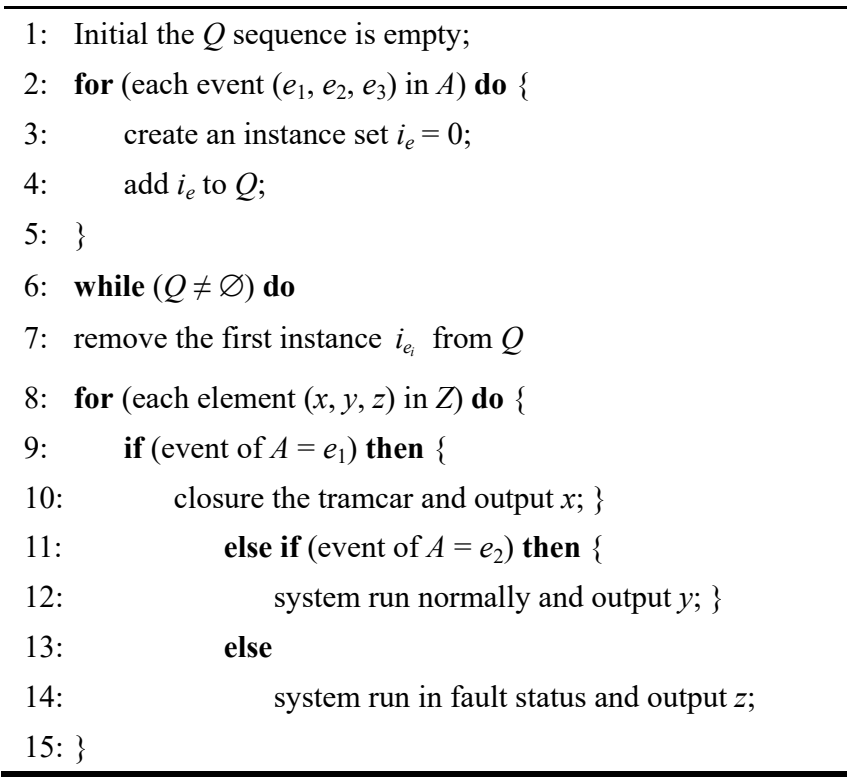

\section{Experimental design and simulation}

In order to certify the validity of scheduling strategy algorithm under the CPS architecture, the open source embedded simulation platform Ptolemy II is adopted, which has multiple different computational models, such as discrete event model (DE), event-oriented model Petra (Feng et al., 2010; Ganesh and Krishna, 2017), finite state machine model (FSM), etc. Since the response of transportation control system mainly depends on external trigger events, these events are generally arranged in the order of time. Therefore, so as to make a better description of the physical process, the adopted Petra computation model can be well interacting with other models and dealing with concurrency in a deterministic way.

Figure 8 Top-level design of a transportation control system (see online version for colours)

DE Director

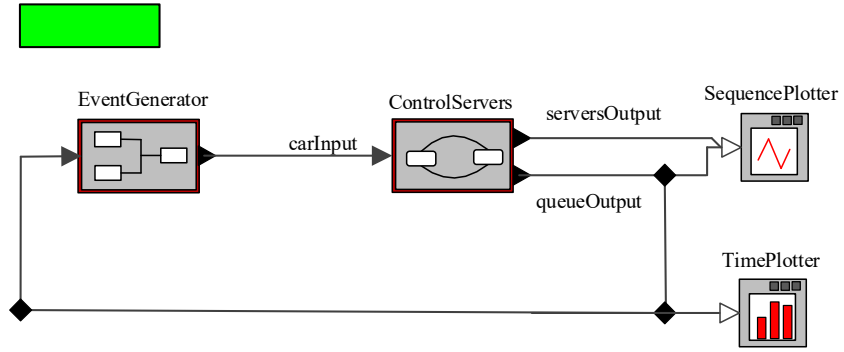

It is worthwhile noting that the operation of transportation control system could be regarded as a single-queue mode. When the external signal occurs, the tramcar is running in one direction and the system provides control services on the principle of first come first serve (FCFS). For example, if there are fewer waiting vehicles in the queue, the control system will put them into the queue to improve transport efficiency. The arrival interval time and control time of tramcar are generated by a random program, during the actual production process, the DE model could be utilised to separate the arrival model and control model of the tramcar, the overall design of control system is shown in Figure 8.

As shown in Figure 9, the composite actor EventGenerator is designed by using hierarchical thought, and the parameter $\min =1$ represents it has existed one vehicle in sequence at least. Actor update is also hierarchically designed and realised as FSM internally, besides there are three processes are defined: block, run and fault, when trigger events occurred, the control strategy will be delivered to actor Init by using immediate transfer action. When the value of the arrival vehicle in the queue is 4 , the control system will step in to block status, and the output will be terminated.

Figure 9 Implementation and design for EventGenerator (see online version for colours)

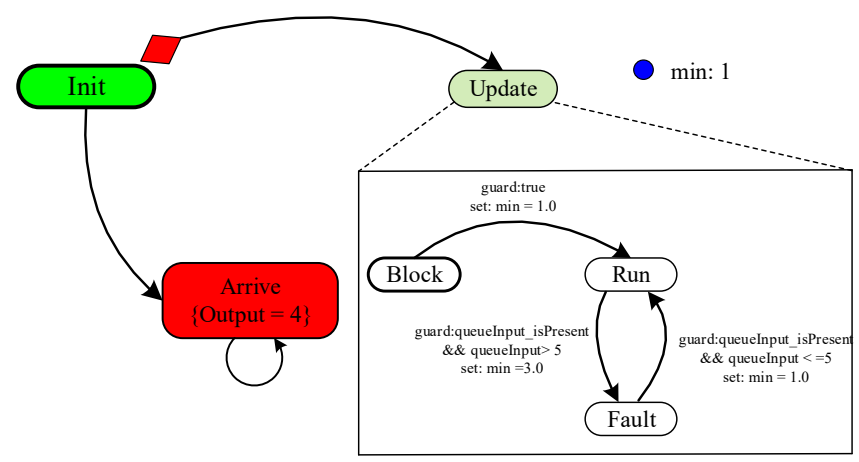

Because of the complexity of the designed model could be reduced and improved by utilising composited design principle that is combining all calculation models and let them have their own hierarchy. As shown in Figure 10, for the designation of composite actor ControlSequence, the initial value is 3 in actor Services. And the output conditions of queue constrained by Enter, Start and Leave sub-actors respectively. Particularly, the transition relationship between them is connected by setting value of guard.

Figure 10 Implementation and design for ControlServers (see online version for colours)

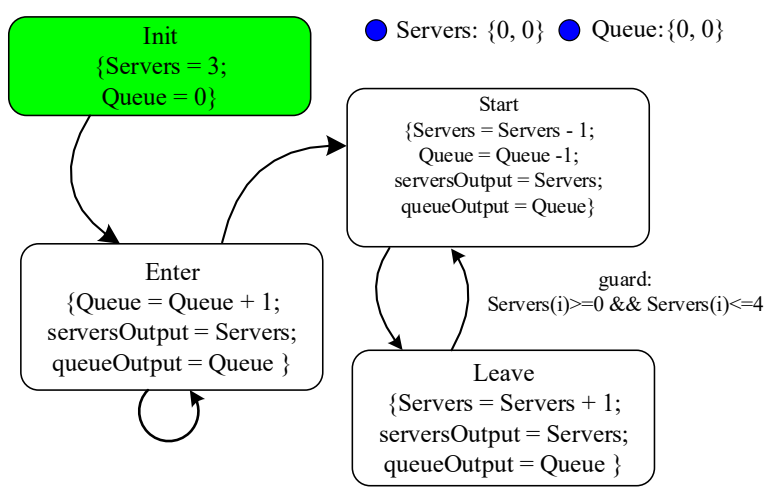


As shown in Figure 11, the actor SequencePlotter shows the number of waiting vehicles sequence (blue) and available servers (red), it can be seen that with time goes by, the blocked vehicles are maintained at 1 , and the waiting sequence showed downtrend. In Figure 12, the HistogramPlotter actor shows the change of waiting vehicles in service queue under the control system over time. At corresponding time 5.5, the waiting vehicles in the queue reach the maximum 117, and with the ControlSequence actor continue operating, the waiting vehicles gradually decreasing, indicating that the control strategy works and has achieved good results.

Figure 11 Waiting sequences (blue) and available servers (red) (see online version for colours)

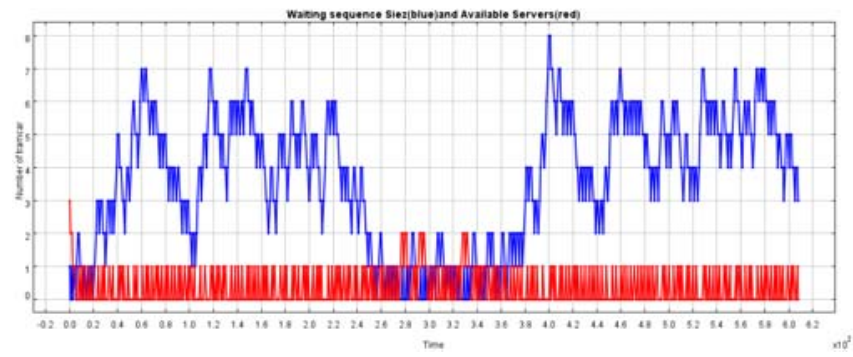

Figure 12 Number of waiting for vehicle in the service queue

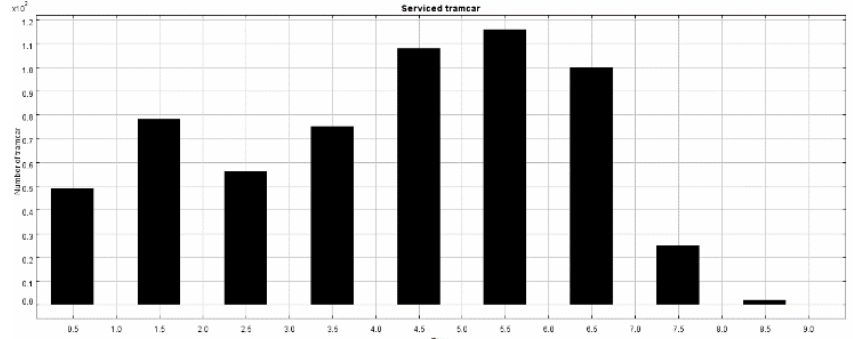

In order to make further verification, compared with the hybrid genetic algorithm (HGA) and the fuzzy clustering scheduling algorithm (FCA), the simulation experiments of execution time under different request tasks are performed. The comparison results are shown in Figure 13.

Figure 13 Comparison of the execution time of the three algorithms under different request tasks

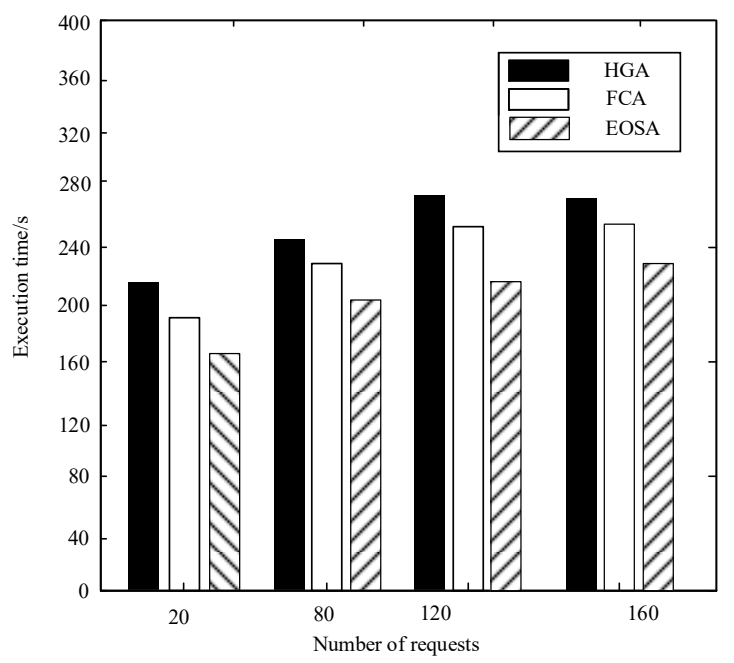

With the number of request tasks increased, the cost of the execution time of three algorithms is also raised. However, the average time of the event-oriented scheduling algorithm (EOSA) is relatively lower. This is because that the event of the service queue has been hierarchically planned, which achieves overall task scheduling load balancing.

\section{Application and conclusions}

By selecting a mine inclined lane transportation control system as an application scenario, the algorithm designed in this paper was applied to the optimisation scheduling scheme. After analysing the partial data of the production report of Table 4, we can conclude that the scheduling algorithm can improve the mine transportation efficiency and realise the dynamic management of production. Therefore, the scheduling scheme has great and realistic significance for establishing an efficient production management system and promoting the long-term development for coal enterprises.

Table 4 Production report of a coal mine on March 2018

\begin{tabular}{lccc}
\hline Label & $\begin{array}{c}\text { Work area } \\
\left(\mathrm{m}^{2} / \mathrm{d}\right)\end{array}$ & $\begin{array}{c}\text { Daily output } \\
(\mathrm{t} / \mathrm{d})\end{array}$ & $\begin{array}{c}\text { Growth rate } \\
(\%)\end{array}$ \\
\hline 1 & 110 & 125 & $4.0 \%$ \\
2 & 130 & 133 & $6.0 \%$ \\
3 & 120 & 142 & $6.8 \%$ \\
4 & 140 & 150 & $7.1 \%$ \\
5 & 130 & 165 & $10 \%$ \\
\hline
\end{tabular}

Since the CPS is a self-knowledge system with multiple levels of information systems and physical systems, and it is a new type system that integrates a large number of sensing devices, computing equipment, and communication networks. These various features make it more suitable for daily production and management of mine enterprises. By analysing the process of inclined lane transportation in coal mine system, we propose a non-memory continuous time model and synchronous response model. In addition, combining relevant event-oriented computational models under discrete domains, the multi-task scheduling algorithm for inclined lane transportation system is developed and implemented. Simulation and experimental results reveal that the proposed scheduling strategy can meet the daily production scheduling tasks and improve the efficiency of transportation.

\section{Acknowledgements}

This research was financially supported by the following funds: The National Nature Science Foundation of China (61170060 and 51874010), the Mine IoTs R\&D Team of Major Innovation Platform and Innovative Talents Team (2017A053) and the Open Project of Collaborative Innovation Center for Industrial Energy Saving and Power Quality Control in Anhui Province (2014051949082861). 
The authors are also grateful to the anonymous reviewers for their constructive comments.

\section{References}

Akkaş, M.A. (2016) 'Using wireless underground sensor networks for mine and miner safety', Wireless Networks, Vol. 24, No. 1, pp.17-26.

Akkaya, I., Derler, P., Emoto, S. and Lee, E.A. (2016) 'Systems engineering for industrial cyber-physical systems using aspects', Proceedings of the IEEE, Vol. 104, No. 5, pp.997-1012.

Bessani, M., Fanucchi, R.Z., Delbem, A.C.C. and Maciel, C.D. (2016) 'Impact of operators' performance in the reliability of cyber-physical power distribution systems', IET Generation Transmission and Distribution, Vol. 10, No. 11, pp.2640-2646.

Chen, N.C., Zhang, X. and Wang, C. (2015) 'Integrated open geospatial web service enabled cyber-physical information infrastructure for precision agriculture monitoring', Computer and Electronics in Agriculture, Vol. 111, pp.78-91, DOI: 10.1016/j.compag.2014.12.009.

Chen, W.H, An, J.Y, Li, R.F., Li, F., Xie, G.Q., Bhuiyan, M.Z.K.A. and Li, K.Q. (2018) 'A novel fuzzy deep-learning approach to traffic flow prediction with uncertain spatial-temporal data features', Future Generation Computer Systems, Vol. 89, pp.78-88, DOI: 10.1016/j.future.2018.06.021

Digalwar, M., Gahukar, P., Raveendran, B.K. and Mohan, S. (2017) 'Energy efficient real-time scheduling algorithm for the mixed task set on multi-core processors', International Journal of Embedded Systems, Vol. 9, No. 6, pp.523-534.

Fang, W.J., Zhang, H., Li, K.C., Zhang, S.X., Marino, M.D. and Jiang, H. (2018) 'An efficient algorithm for modelling and dynamic prediction of network traffic,' International Journal of Computational Science and Engineering, Vol. 16, No. 3, pp.311-320.

Feng, T.H., Lee, E.A. and Shruben, L.W. (2010) 'Ptera: an event-oriented model of computation for heterogeneous systems', Proceedings of 10th ACM International Conference on Embedded Software, Arizona, USA, Vols. 24-29, pp.219-228.

Ganesh, M.S. and Krishna, V. (2017) 'Improving the performance by message broadcasting in VANETS', International Journal of High-Performance Computing and Networking, Vol. 10, No. 3, pp.194-200.

Gonçalves, F.S., Raffo, G.V. and Becker, L.B. (2016) 'Managing CPS complexity: design method for unmanned aerial vehicles', IFAC-PapersOnLine, Vol. 49, No. 32, pp.141-146.

Gu, Z.H., Li, H., and Cai, H.B. (2016) 'Improving computation efficiency of schedulability of transaction-based task model for distributed real-time embedded systems,' International Journal of Embedded Systems, Vol. 8, No. 4, pp.277-292.

Isasa, J.A.E., Larseb, P.G. and Hansen, F.O. (2017) 'A holistic approach to energy-aware design of cyber-physical systems', International Journal of Embedded Systems, Vol. 9, No. 3, pp.283-295.

Jindai, V. and Bedi, P. (2018) 'An efficient algorithm for modeling and dynamic prediction of network traffic', International Journal of Computational Science and Engineering, Vol. 16, No. 4, pp.390-400.
Khan, M., Li, S., Wang, Q.X. and Shao, Z.L. (2016) 'CPS oriented control design for networked surveillance robots with multiple physical constraints', IEEE Transactions on Computer-Aided Design of Integrated Circuits and Systems, Vol. 35, No. 5, pp.778-791.

Lee, J. and Kang, G.S. (2017) 'Development and use of a new task model for cyber-physical systems: a real-time scheduling perspective', Journal of Systems and Software, Vol. 126, pp.45-56, DOI: 10.1016/j.jss.2017.01.004.

Li, J.Z., Ren, P and Yang, D.Y. (2017) 'Study on perception and control layer of mine CPS with mixed logic dynamic approach', Proceedings of 7th International Conference on Electronics and Information Engineering, Nanjing, China, Vols. 17-18, pp.322-331.

Lin, J., Yu, W., Yang, X.Y., Yang, Q.Y., Fu, X.W. and Zhao, W. (2016) 'A real-time en-route route guidance decision scheme for transportation-based cyber-physical systems', IEEE Transactions on Vehicular Technology, Vol. 66, pp.2551-2566.

Liu, H., Sun, D.H. and Liu, W.N. (2016) 'Lattice hydrodynamic model based traffic control: a transportation cyber-physical system approach', Physica A: Statistical Mechanics and Its Applications, Vol. 461, pp.795-801, DOI: 10.1016/j.physa.2016.06.069

Liu, Y., Lin, M., Taniguchi, I.T. and Tomiyama, H.Y. (2017) 'A dual-mode scheduling approach for task graphs with data parallelism', International Journal of Embedded Systems, Vol. 9, No. 2, pp.277-292.

Muller, H.A. (2017) 'The rise of intelligent cyber-physical systems', Computer, Vol. 50, No. 12, pp.7-9.

Nayak, A., Reyes, R.L., Lee, S. and Nof, S.Y. (2016) 'Resource sharing in cyber-physical systems: modeling framework and case studies', International Journal of Production Research, Vol. 54, No. 23, pp.6969-6983.

Plaza, A.M., Díaz, J. and Pérez, J. (2018) 'Software architectures for health care cyber-physical systems: a systematic literature review', Journal of Software Evolution and Process, Vol. 30, No. 7, pp.1-23.

Rao, A., Carreón, N., Lysecky, R. and Rozenblit, L. (2018) 'Probabilistic threat detection for risk management in cyber-physical medical systems', IEEE Software, Vol. 35, No. 1, pp.38-43.

Rossi, M., Rizzon, L., Passerone, R., Minakov, I., Sartori, D. and Brunelli, D. (2017) 'Non-invasive cyber-physical system for data center management', Sustainable Computing Informatics \& Systems, Vol. 16, pp.66-75, DOI: 10.1016/j.suscom.2017.09.003.

Sadreazami, H., Mohammadi, A. and Asif, A. (2018) 'Distributed graph-based statistical approach for intrusion detection in cyber-physical systems', IEEE Transactions on Signal and Information Processing Over Networks, Vol. 4, No. 1, pp.137-147.

You, M., Liu, Q.T. and Sun, H.J. (2017) 'New communication strategy for spectrum sharing enabled smart grid cyber-physical system', IET Cyber-Physical Systems: Theory \& Applications, Vol. 2, No. 3, pp.136-142.

Zhang, Y., Qiu, M., Tsai, C.W. and Hassan, M.M. (2017) 'Health-CPS: healthcare cyber-physical system assisted by cloud and big data', IEEE Systems Journal, Vol. 11, No. 1, pp.88-95. 